\title{
Schematic Design of Move-in-mud Robot with Double Spiral Blade
}

\author{
Bingbing Yan ${ }^{\mathrm{a}}$, Baolin Yin ${ }^{\mathrm{b}}$, Wenbo Ren ${ }^{\mathrm{c}}$ and Yunming Du ${ }^{\mathrm{d}}$ \\ ${ }^{1}$ School of Mechanical and Electronic Engineering, Jiamusi University, Jiamusi 154007, China \\ ayanbingbing@126.com, b499677887@qq.com, cyinblin@163.com, dduyunming@126.com
}

Corresponding author: Baolin Yin

Keywords: Move-in-mud Robot, schematic design, spiral blade

\begin{abstract}
The move-in-mud robot is a self-supporting type of special equipment working in soil. The existing literature shows that the key issues of smooth working of the move-in-mud robot are that it should have the stable supporting conditions and reliable steering function. However, the test results of the existing experimental prototype are not ideal, and there is more or less the slip phenomenon. In this paper, a new type of move-in-mud robot with double spiral blades is presented to solve the above problem. The front and rear spiral blades are connected by the steering joint with 2PSP/S parallel mechanism, and a tapered head is equipped to assist attacking mud. The friction between the rotating spiral blades in the soil and the soil can generate the axial force to drive the robot going forward. Meanwhile, the steering joint is used to achieve adjusting the forward direction of the robot within a small range. In addition, the supporting connection mechanism of steering joint is a good solution to the mutual winding problem. In short, the above schematic design of move-in-mud robot with double spiral blade provides a new solution for the engineering practice of move-in-mud robot.
\end{abstract}

\section{Introduction}

The excavating whole work under water is a key process of the salvaging sunken ships, which requires diver dives into the water. In order to instead of diver to finish this dangerous work, a special robot is proposed by researchers, namely move-in-mud robot [1]. According to available records, now the basic research has studied by only some domestic universities and research institutes (such as the Harbin Engineering University, Chinese Academy of Science, Shenyang Institute of Automation, Wuhan University of Science and Technology, Northwestern Polytechnic University, etc.)[2, 3, 4, 5, 6], which mainly concentrated on the scheme design, kinematics modeling and simulation, motion control and position detecting and soil mechanics modeling, geometric modeling, etc.[7, 8].

The above research results shown that all of the existing move-in-mud robot were designed based on the peristalsis principle of earthworm, its structure was consist of the creeping mechanism, supporting mechanism and steering joint, which can realize the peristaltic forward of move-in-mud robot by the cyclical alternating movements. The movement principle diagram of the robot is shown in Figure 1. Through the comparative analysis of the existing literatures we known that the creeping movement of the move-in-mud robot is designed based on the peristalsis principle of earthworm needs radial expansion of the supporting mechanism cyclically, but the intensity of soil is destroyed in a certain extent after radial expansion many times, which results in the insufficient supporting force for the move-in-mud robot and causes the backward slip phenomenon. Therefore, to find a reasonable mechanical structure of move-in-mud robot to solve the slip phenomenon has an important significance nowadays.

\section{The Overall Design Scheme}

Aim at the insufficient support force problem for the existing move-mud-robot design, a new type of move-in-mud robot with double spiral blades is proposed in the paper based on the spiral propulsion theory; its structure diagram is shown in Figure 2. In this scheme, the double spiral blade (left and right) and the steering joint with 2PSP/S parallel mechanism in series are used for the robot 
to go forward and turn, at the same time, a tapered head is equipped to assist attacking mud. When the double spiral blades reverse rotating, the friction between the blade and the soil can produce the axial force, which can eliminate the effect of torque and provide stable support condition. As you can see from Figure 1, the outside diameter of the rear spiral blade is larger than that of the front, its purpose is to provide a greater driving force for robot. In addition, the steering joint with 2PSP/S parallel mechanism can be used to adjust the forward direction of robot within a small range.

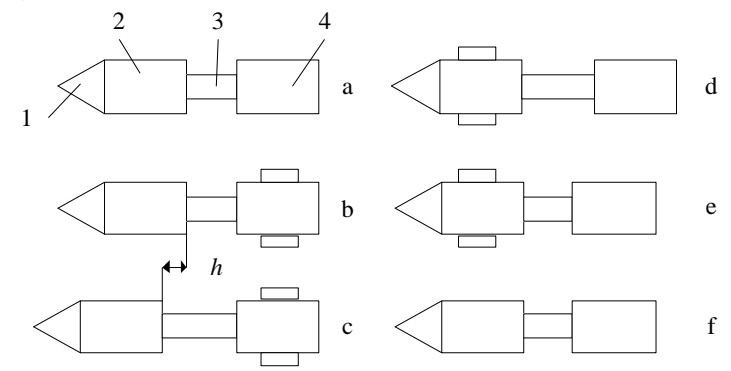

1-mud tapping mechanism, 2-front supporting mechanism 3-steering joint mechanism, 4-back supporting mechanism

Fig. 1 Movement Principle Diagram of the Move-in-mud Robot

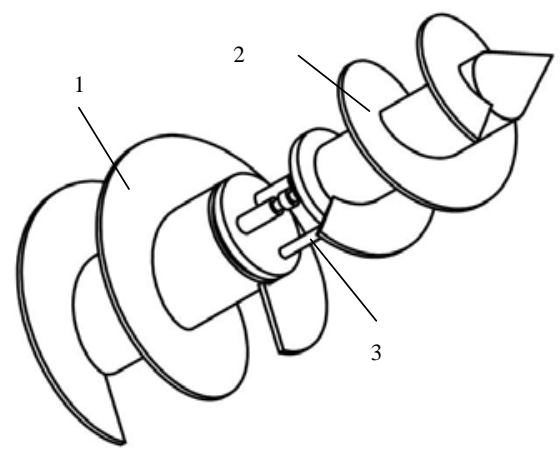

1- Left Spiral Blade, 2- Right Spiral Blade, 3- Steering Joint

Fig. 2 Structure Diagram of Move-in-mud Robot with Double Spiral Blade

\section{Steering Joint with 2PSP/S Parallel Mechanism}

Compared with the working environment of existing industrial robots and intelligent robots, the soil environment has a high degree of complexity and uncertainty, so this requires that the move-in-mud robot should have the strong power and non-redundant output and simple control system. In this scheme, the 2PSP/S parallel mechanism is adopted as the steering joints so as to achieve adjusting the forward direction of the robot within a small range. This steering joint consist of the base, the movable platform and three motion branch chains, and its structure diagram is shown in Figure 3.

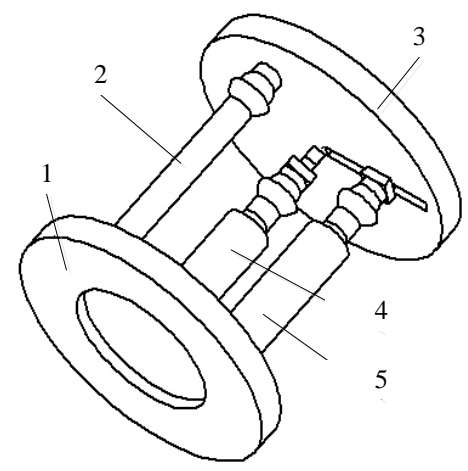

1- Base, 2-First Motion Branch, 3-Movable Platforms, 4-Second Motion Branch, 5-Third Motion Branch

Fig. 3 2PSP/S Parallel Mechanism 
The base and movable platform are connected by three motion branch chains. Among them, the first branch chain consist of a rigid rod and a spherical pair, and the end of this rod is fixed with the base, the other end is connect with the movable platform by a spherical pair. The second and third branch chain have the same of structure, both of them consist of hydraulic cylinders and spherical pairs, and the end of hydraulic cylinder is fixed with the base, and the push rod of hydraulic cylinder is connected by a spherical pair that installed inside the slide of the movable platform. This mechanism has two rotational degrees of freedom, which can ensure the robot turn within a small range just need to adjust the length of the push rod of the two hydraulic cylinders. Diagram of angle adjustment of the movable platform in the three different forms is shown in Figure 4.

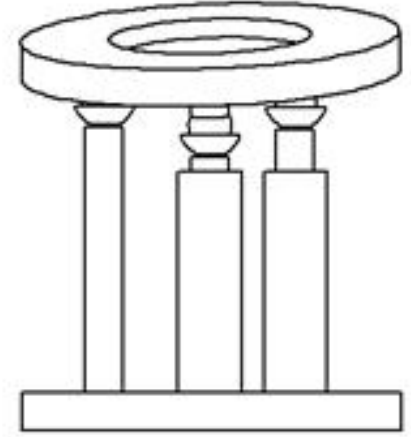

(a)

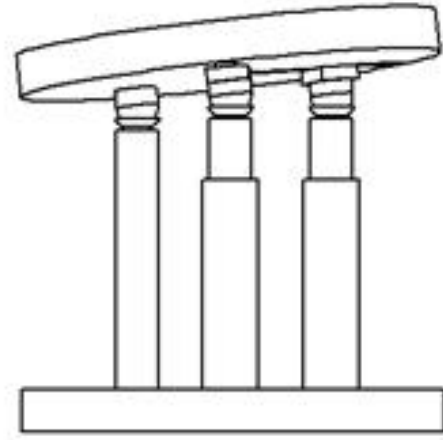

(b)

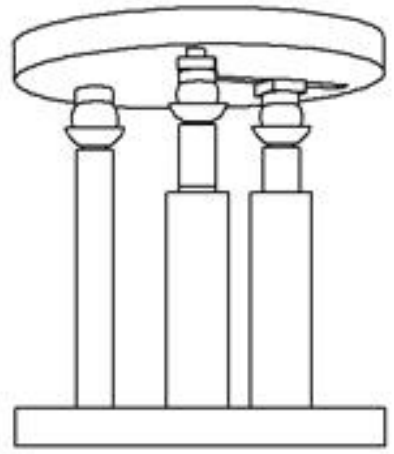

(c)

(a) Horizontal Angle between the Plane (b) Vertical Angle between the Plane (c) Composite Angles Fig. 4 Angle Adjustment of the movable platform

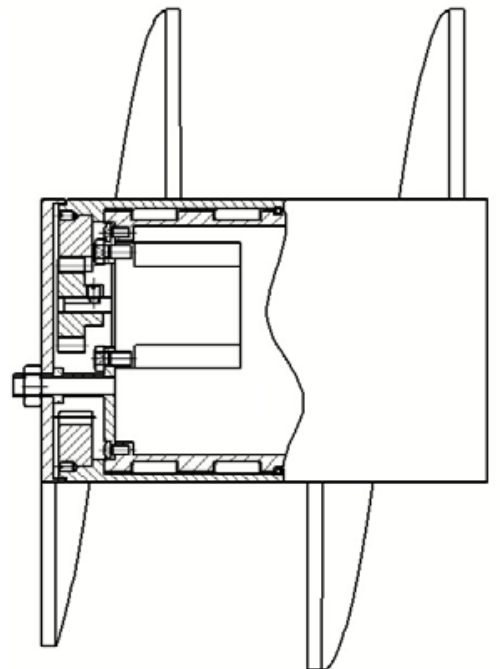

1-Hydraulic Motor, 2-End Cap, 3-Inner Sleeve, 4-Ring Gear, 5- Outer sleeve, 6-Roller, 7- Gear, 8Base (or Movable Platform)

Fig. 5 Steering Joint Supporting Connecting Mechanism

\section{Supporting Connection Mechanism of Steering Joint}

To sum up, we know that the power for the movement of robot in the soil is generated by friction between the double spiral bladed and the soil, and the reverse rotation movement of before and rear spiral blades is driven by the hydraulic motor is assembled in the internal body. This may cause the mutual winding phenomenon of power source line distributed in the internal body. The design of supporting connection mechanism of steering joint is put forward to solve this problem (see Figure 5). This mechanism is mainly composed of the hydraulic motor, the end cap, the inner sleeve, the ring gear, the outer sleeve, the roller, the gears, etc. Through bolts, the hydraulic motor and the inner sleeve are fixed at one end of the inner end cap, and the other end is connected with the base (or movable platform) by the positioning shaft shoulder and a locking nut. Furthermore, the outer sleeve is nested on the inner sleeve by kingpins, and the spiral blade is welded on the outer wall of the outer 
sleeve. When the hydraulic motor drives the outer sleeve rotating, the inner sleeve and the base (or movable plate) is not rotated. Meanwhile, the ring gear fixed by pins to the inner wall of the outer sleeve engages with the gear, so that the spiral blades can be rotated along its own axis. Based on the above design, under the premise of its fixed on the base (or movable plate), the output torque of the hydraulic motor can drive the spiral blade rotating around the central axis of the robot through the gear meshing between the gear and the ring gear. At the same time, the power lines can go through the hole of the inner cover so as to solve the mutual winding phenomenon.

\section{Summary}

(1) Through analyzing the existing research results of move-in-mud robot based on the creeping principle, a schematic design of move-in-mud robot with double spiral blade is proposed based on the spiral propulsion theory, and the way of spiral propulsion is adopted instead of radial supporting of peristalsis, to a certain extent, which can overcome the problem of insufficient support force, so the slip phenomenon of move-in-mud robot should can be avoided in the experiment.

(2) In view of the complexity and uncertainty of the soil environment, a steering joint with 2SPS/S type parallel mechanism is proposed to ensure the strong power output and flexible steering control. And the mutual winding problem of power source line is also solved by the supporting connection mechanism of steering joint, which can provide the basis for the engineering practice of move-in-mud robot.

\section{Acknowledgment}

The research was supported by the Key Project of Chinese Ministry of Education. (212042), the Natural Science Fund of Heilongjiang Province (E201254), and the New Century Excellent Talents In Heilongjiang Provincial University (1252-NCET-021). The authors would like to thank their supporting.

\section{References}

[1]. S.L Feng, J.H. Wang, Y. Lin, B. Sun. Ship Engineering. Vol. 25 (2003) No. 5, p. 32-34.

[2]. F.J Ren, B.B Yan. Chinese Journal of Mechanical Engineering. Vol. 45 (2009) No. 1, p. 50-55.

[3]. H.X. Wei, Q.X. Meng, T.M. Wang. Shipbuilding of China. Vol. 44 (2003) No. 160, p. 89-92.

[4]. S.L. Feng, J.H. Wang, Y. Lin, B. Sun. Ship Engineering. Vol. 25 (2003) No. 5, p. 32-34.

[5]. Y. Zhang. Ship \& Ocean Engineering. Vol. 36 (2007) No. 6, p. 100-102.

[6]. Y. Zhang, H. Sun, Y. Hu. Journal of Wuhan Automotive Polytechnic University. Vol. 27 (2005) No. 4, p. 43-46.

[7]. D.J Wang, S.J. Liu, L. Xue, F.J. Ren, Q.X. Meng. Journal of Harbin Institute of Technology. Vol. 41 (2009) No. 1, p. 94-97.

[8]. Q.X. Meng, Z.L. Zhang, Z. Wang, L.W. Cao, X. Sun. Mechanical Science and Technology. Vol. 25 (2006) No. 8, p. 94-97. 